\title{
LA PUBLICATION \\ DU TREATISE ON ELECTRICITY AND MAGNETISM DE JAMES CLERK MAXWELL
}

\author{
Franck ACHARD
}

RÉsumé : Cet article vise à éclairer le contexte universitaire et éditorial qui favorisa la publication du Treatise on electricity and magnetism de James Clerk Maxwell afin de mieux cerner la nature de cette entreprise scientifique. Le projet fut formé en 1867 à l'occasion d'une réforme introduisant l'étude de l'électricité et du magnétisme dans l'enseignement délivré à Cambridge et s'inscrivait dans un mouvement plus vaste qui développait l'enseignement de ces disciplines dans les universités britanniques. L'étude des relations entre le projet de Maxwell et d'autres ouvrages contemporains indique que le Treatise était en situation de devenir un ouvrage de référence en Grande-Bretagne. Ainsi, le livre de Maxwell était-il l'instrument d'une très ambitieuse tentative de transformation de l'électromagnétisme, indissociable de son contexte et conforme à sa fonction pédagogique.

Mors-clés: Treatise on electricity and magnetism, Maxwell, publication scientifique, électromagnétisme, université de Cambridge.

ABSTRACT: This paper seeks to clarify the academic and publishing background which promoted the publication of James Clerk Maxwell's Treatise on electricity and magnetism and tries to pinpoint the nature of this scientific enterprise. The project was formed in 1867 when a reform introduced the study of electricity and magnetism in the courses offered at Cambridge. It was part of a wider movement which generalized the teaching of these subjects in British universities. The study of the links between Maxwell's project and other contemporaneous books shows that the Treatise was about to become a reference work in Britain. Thus Maxwell's book was the instrument of a very ambitious attempt to transform electromagnetism, which must be related to its background and to its teaching function.

KEYworDs : Treatise on electricity and magnetism, Maxwell, scientific publication, electromagnetism, Cambridge University.

Revue de synthèse : $4^{\circ}$ S. $n^{\circ} 4$, oct.-déc. 1998, p. 511-544. 
ZUSAMMENFASSUNG : In diesem Artikel wird gezeigt, welche universitären und verlagstechnischen Umstände das Erscheinen von Maxwells Treatise on electricity and magnetism begleitet haben. Der Plan für dieses Buch geht auf Jahr 1867 zurück, als infolge einer Universitätsreform Elektrizität unf Magnetismus in Cambridge und an anderen britischen Universitäten in den Lehrplan aufgenommen wurden. Der Vergleich mit anderen Büchern über diese Themen zeigt, daß Maxwells Treatise sehr schnell das einschlägige Standardwerk in Großbritannien geworden ist. Aus dem Kontext seiner Entstehung und seiner pädagogischen Funktion wird verständlich, warum das Buch ein wirksames Instrument zur Neuformulierung von Elektrizität und Magnetismus geworden ist.

STICHWÓRTER : Treatise on electricity and magnetism, Maxwell, wissenschaftliche Veröffentlichung, Elektromagnetismus, Universität Cambridge.

Franck Achard, né en 1967, ingénieur en informatique, achève un doctorat d'histoire des sciences à l'École des hautes études en sciences sociales de Paris. Sa thèse porte sur les conditions intellectuelles et institutionnelles de l'élaboration du Treatise on electricity and magnetis.: de James Clerk Maxwell.

Adresse : EHESS/Centre Alexandre-Koyré, Muséum national d'histoire naturelle, Pavillon Chevreul, 57 rue Cuvier, 75231 Paris Cedex 05. 
Le Treatise on electricity and magnetism du physicien écossais James Clerk Maxwell (1831-1879), paru en 1873, est sans doute, après les Principia de Newton, un des livres les plus célèbres de l'histoire de la physique. C'est en effet ce texte qui fit connaître la théorie du champ électromagnétique dont on connaît l'influence considérable sur les recherches électromagnétiques de la fin du XIX ${ }^{e}$ siècle et, à travers elles, sur l'ensemble de la physique du $x x^{e}$ siècle. On peut citer parmi les aspects les plus marquants de cette théorie le fait qu'elle décrivait pour la première fois la lumière comme un phénomène électromagnétique et qu'elle fut à l'origine de la télégraphie sans fil.

Il est également bien connu que Maxwell rédigea l'essentiel de son ouvrage au cours d'une période où il s'était partiellement retiré de la vie universitaire. En février 1865, il avait en effet démissionné de son poste de professeur de philosophie naturelle au King's College de Londres et avait passé la majeure partie des six années suivantes dans sa résidence écossaise de Glenlair sans occuper de poste universitaire. Ce n'est qu'en mars 1871 que Maxwell mettait fin à cette retraite pour occuper la chaire de physique expérimentale nouvellement créée à Cambridge. Notre principale source sur la vie de Maxwell est la biographie publiée par son ami d'enfance, le révérend Lewis Campbell, deux ans après sa mort. Les conditions de la rédaction du Treatise y sont relatées de la façon suivante:

"The years which followed the resignation of his post at King's College were spent, for the most part, at Glenlair [...] And Manwell took advantage of this retirement to embody some of the results of his investigations in substantive books. The great work on Electricity and magnetism, although not published till 1873, was now taking definitive shape, and the treatise on Heat, which appeared in 1870 [sic], had been undertaken as a by-work during the same period ${ }^{1}$."

Ces quelques lignes semblent suggérer, sans le dire explicitement, que la rédaction du Treatise fut entreprise dès l'installation de Maxwell à Glenlair en 1865. Certains historiens ont même supposé que ce projet fut l'une des principales motivations de la démission de Maxwell de son poste à Londres $^{2}$. En janvier 1865, Maxwell avait en effet publié un de ses articles majeurs en électromagnétisme, «A dynamical theory of the electromagne-

1. Pour plus de précisions sur les références citées en notes se reporter à la bibliographie finale, p. 542-544. Ici, CAmpBell \& GarneTt, 1882, p. 320. L'ouvrage de Maxwell sur la chaleur, Theory of heat, fut en fait publié en 1871.

2. Voir, par ex., EveriTt, 1978, p. 199, et HENDRY, 1986, p. 221. 
tic field », qui, sur le plan mathématique, contenait déjà l'essentiel de la théorie qu'il allait présenter dans son traité. Il est donc tentant d'en déduire que Maxwell fut animé du désir de diffuser plus largement sa théorie dès 1865 , à un moment où il l'estimait suffisamment aboutie.

Un tel récit est cependant révélateur du fait que les conditions de la publication de l'ouvrage ne sont généralement que très succinctement envisagées. Le projet d'écrire un traité y apparaît comme uniquement déterminé par le cheminement intellectuel de Maxwell et l'état de maturité de sa théorie. Il néglige totalement, en revanche, le rôle que joua l'environnement social et institutionnel dans la naissance du projet de Maxwell. Il ne se demande pas, par exemple, si le contexte était ou non favorable à la publication d'un vaste traité sur l'électricité et le magnétisme. En termes économiques, on pourrait dire que le récit traditionnel explique la production du Treatise par l'offre, c'est-à-dire la théorie de Maxwell arrivée à maturité, mais oublie de prendre en compte la demande, c'est-à-dire l'existence de lecteurs susceptibles de lire le livre ${ }^{3}$. Cette représentation du savant édifiant sa théorie dans la solitude est bien symbolisée, dans la biographie de Campbell, par l'image de Maxwell écrivant son traité dans sa demeure de Glenlair, perdue dans la campagne écossaise.

Sans contester l'importance des motivations intellectuelles de Maxwell dans la naissance du Treatise, le propos est ici d'éclairer le contexte institutionnel qui favorisa sa publication. L'étude se compose de quatre parties. La première décrit les étapes de la production du traité. La correspondance de Maxwell, éditée récemment par Peter M. Harman, permet d'établir une chronologie précise de la production du Treatise. Les archives de l'Oxford University Press, éditeur du traité, complètent notre connaissance du processus de la publication. Ces documents indiquent, en particulier, que Maxwell entama la rédaction de son ouvrage, non pas dès 1865 , mais vers la fin de l'année 1867. La seconde partie s'attache à montrer que la publication du Treatise fut favorisée par un mouvement de réforme de l'enseignement scientifique en Grande-Bretagne qui se développa autour de cette même année 1867. Plus précisément, la publication d'un traité sur l'électricité et le magnétisme était rendue nécessaire par une réforme de l'enseignement de la physique à Cambridge entre 1867 et 1871 qui introduisit ces sujets dans les examens du Mathematical Tripos et aboutit à la création de la chaire de physique expérimentale qu'allait occuper Maxwell. Il s'avère également que ce dernier, loin d'être totalement isolé du monde universitaire, a joué un rôle important dans la réforme à Cambridge. La troisième partie étudie la place du Treatise dans son contexte éditorial et ses relations

3. Graeme Gooday a employé les critères d'offre et de demande dans son analyse des conditions d'apparition des laboratoires de physique en Grande-Bretagne. Voir GoodAY, 1990, p. 26. 
avec d'autres ouvrages contemporains. Elle montre que les conditions de sa publication le destinaient à occuper la place d'un ouvrage de référence sur l'électricité et le magnétisme dans le monde scientifique et universitaire de langue anglaise. Enfin, la quatrième partie s'appuie sur ces différents éléments pour proposer une nouvelle image de l'entreprise scientifique que Maxwell entendait réaliser à travers son traité. Elle suggère alors de nouvelles perspectives pour l'étude du Treatise, partant du principe que le sens que Maxwell et ses lecteurs contemporains attribuaient à l'ouvrage ne doit pas être dissocié des conditions de sa production et de sa circulation.

\section{I. - LE PROCESSUS DE PUBLICATION DU TREATISE}

Les documents dont nous disposons indiquent que la rédaction du Treatise on electricity and magnetism ne fut pas entreprise avant la fin de l'année $1867^{4}$. Le traité est en effet mentionné dans une lettre datée du 27 novembre de cette année écrite par le professeur de philosophie naturelle à Édimbourg, Peter Guthrie Tait, et adressée à Maxwell. Tait y exprimait la satisfaction d'apprendre la décision que Maxwell avait prise de rédiger un traité sur l'électricité : "I am delighted to hear that you are going to do a Senate-House Treatise on Electricity. The sooner the better ${ }^{5}$." Tait n'avait donc appris ce projet que depuis peu, probablement après la précédente lettre de Maxwell, datée du 13 novembre, qui n'y faisait pas allusion. Cette même lettre du 27 novembre nous apprend, en outre, qu'une rencontre entre les deux hommes avait été prévue à Glasgow mais n'avait pas pu avoir lieu ${ }^{6}$. On peut donc imaginer que, malgré une indisponibilité de Tait, Maxwell ait fait le voyage entre le 13 et le 27 pour y rencontrer l'illustre physicien sir William Thomson (plus tard lord Kelvin), professeur de philosophie naturelle dans cette ville. À cette occasion, Maxwell aurait fait part de ses intentions à Thomson, qui en aurait par la suite informé Tait.

Quoi qu'il en soit, il est certain que Tait et Thomson furent parmi les premiers avertis. Les trois hommes entretenaient, en effet, des relations d'amitié où les échanges scientifiques occupaient une place prépondérante. Maxwell et Tait, tous deux nés en 1831, avaient fréquenté ensemble l'Academy, puis l'université d'Édimbourg. Tait, puis Maxwell deux ans plus

4. Peter Harman, introd., in Maxwell, 1995, p. 24.

5. Maxwell, $1995, \mathrm{n}^{\circ} 276, \mathrm{n}$. 2. Nous reviendrons plus loin sur l'expression de « SenateHouse Treatise" employée par Tait (p. 522).

6. Ibid. : "I was sorry not to meet you at Glasgow.» 
tard, étaient ensuite partis à Cambridge pour préparer le prestigieux examen du Mathematical Tripos ${ }^{7}$ qu'ils avaient tous deux brillamment réussi (Tait se classant premier en 1852 et Maxwell second en 1854). Thomson, second du Mathematical Tripos en 1845, était de huit ans leur aîné. Dès les années 1840 et 1850 , il avait acquis un renom considérable par ses recherches sur l'électrostatique, sur le magnétisme et sur la théorie de la chaleur ${ }^{8}$. Très tôt, il avait joué un rôle de mentor vis-à-vis de Maxwell. C'est en particulier à lui que Maxwell s'était adressé en 1854 pour ordonner ses lectures en vue d'entamer des recherches en électromagnétisme?. Thomson et Tait, enfin, avaient fait connaissance en 1861 au moment de la nomination de ce dernier à la chaire d'Édimbourg et, depuis cette rencontre, préparaient ensemble un vaste Treatise on natural philosophy.

À partir de fin novembre 1867, les allusions au traité de Maxwell revinrent régulièrement dans la correspondance avec ses deux interlocuteurs. Une lettre de Maxwell à Thomson, datée du 20 février 1868, nous indique son intention de proposer son ouvrage à la Clarendon Press, l'organisme de publication rattaché à l'université d'Oxford ${ }^{10}$. Les archives de l'Oxford University Press confirment que la Clarendon Press reçut une offre de Maxwell entre le 18 février et le 10 mars ${ }^{11}$. Le 8 mai, l'instance de décision de la Clarendon Press, le Board of delegates, acceptait définitivement de publier le traité de Maxwell ${ }^{12}$. Ce dernier en informait Tait dans une lettre datée du 3 août ${ }^{13}$.

Nous ne disposons malheureusement pas de la correspondance échangée en 1868 entre Maxwell et la Clarendon Press. Elle serait précieuse pour savoir comment Maxwell présentait alors son projet. Il est toutefois significatif que la Clarendon Press l'ait considéré comme conforme à la politique éditoriale qu'elle avait lancée depuis quelques années. Autour de 1860, l'organisme d'Oxford avait décidé de développer la publication d'ouvrages à vocation pédagogique et avait créé un School Book Committee chargé de définir les besoins les plus pressants. Celui-ci avait notamment souligné le

7. Pour une présentation du Mathematical Tripos, voir p. 520.

8. Pour la carrière de William Thomson, voir SMITH \& WISE, 1989.

9. MAXWELL, $1990, n^{\circ} 45$, p. 237-238.

10. MaXwell, 1995, $n^{\circ} 281$, p. 346. Pour une citation complète, voir p. 534.

11. Une première mention du projet de Maxwell apparaît dans le registre du School Book Committee à la date du 10 mars 1868: «11. Mr Maxwell on Electricity (by Mr Price)/ Mr Price authorized to confer with $\mathrm{MrM}$. with a view to the acceptance of the work. » La précédente réunion du School Book Committee avait eu lieu le 18 février.

12. Voir Oxford University Press Archives (cité par la suite comme OUPA), Order Book, 8 mai 1868 : "It was agreed to accept on the recommendation of the School Book Committee a Mathematical Treatise on Electricity by MrJ Clerk Maxwell, FRS, the details to be settled by the School Book Committee. " L'offre de Maxwell avait précédemment été acceptée par le School Book Committee le 28 avril 1868, voir OUPA, registre du School Book Committee.

13. MAXWELL, $1995, \mathrm{n}^{\circ} 299$, p. 416 : "With respect to my electrical treatise the Clarendon people have I believe accepted it. " 
manque criant d'ouvrages consacrés aux sciences physiques. De nouveaux livres furent donc progressivement publiés dans une collection intitulée "Clarendon Press Series ${ }^{14}$ ". C'est en particulier dans cette collection que furent publiés le premier volume du Treatise on natural philosophy de Thomson et Tait en octobre 1867 et le Treatise de Maxwell en mars 1873. Il est probable que ce sont Thomson et Tait qui conseillèrent à Maxwell de proposer son ouvrage à la Clarendon Press. Peut-être allèrent-ils jusqu'à le «parrainer » directement auprès de l'éditeur. Le principal interlocuteur des auteurs était alors Bartholomew Price, le professeur de philosophie naturelle à l'université d'Oxford, qui avait été nommé secrétaire général de la Clarendon Press en $1868^{15}$. C'est sans doute ce dernier qui joua un rôle prépondérant dans l'accord de la Clarendon Press.

La correspondance de Maxwell avec Tait et Thomson fournit également de nombreuses informations sur la rédaction du Treatise. À l'encontre du récit traditionnel qui tend à laisser croire que Maxwell a écrit son traité dans un isolement quasi total, il apparaît que ces échanges épistolaires étaient fort utiles à l'élaboration de l'ouvrage. Les deux interlocuteurs y servaient à la fois de sources d'information et de premiers lecteurs. Maxwell y exposait brièvement certaines de ses démonstrations mathématiques ou de ses innovations théoriques, demandait à Thomson de confirmer des positions qu'il lui attribuait ou s'enquerait de l'origine de certains théorèmes. Les sujets scientifiques abordés par Maxwell dans ses lettres correspondaient donc approximativement à ceux sur lesquels il écrivait au même moment. On trouve, de plus, quelques remarques explicites sur l'état d'avancement de sa rédaction. Ces éléments ont permis récemment à Harman de reconstituer la chronologie de la rédaction ${ }^{16}$. Il suffit pour notre propos de noter que celui-ci écrivit probablement l'essentiel des trois premières parties entre la fin de l'année 1867 et le début de 1870 . À cette période, il entama la rédaction de son livre sur la chaleur, le Theory of heat, et poursuivit parallèlement l'écriture de la quatrième partie.

Entre juin 1868 et le début 1870, Maxwell n'échangea aucune correspondance avec la Clarendon Press ${ }^{17}$. Comme nous venons de le voir, cette période fut consacrée à la rédaction de la majeure partie de l'ouvrage et Maxwell ne jugeait, semble-t-il, pas utile d'informer la maison d'édition des progrès de sa rédaction. Au début de 1870 , il estimait donc que son tra-

14. SuTCLFFE, 1978 , p. 16-24.

15. Sur le rôle prépondérant de Price à la Clarendon Press, voir Sutcuffe, 1978, p. 27-33.

16. MAXwELt, 1995, introd., p. 24-32.

17. Les archives de l'Oxford University Press contiennent des registres, appelés Letters Book, contenant des copies de toutes les lettres envoyées par la Clarendon Press à ses collaborateurs à partir du 2 juin 1868. La première lettre adressée à Maxwell dont nous disposons porte la date du 19 février 1870 . 
vail était suffisamment avancé pour reprendre contact avec Price et préparer l'impression de l'ouvrage ${ }^{18}$. Toutefois, malgré des demandes répétées de Price, il ne donna son accord pour entamer l'impression qu'à la fin de l'année ${ }^{19}$. Entre-temps, Maxwell transmit la première partie de son manuscrit $^{20}$ pour permettre à Price de faire quelques recommandations concernant la mise en forme du livre (taille de la page ${ }^{21}$, numérotation des articles et des équations ${ }^{22}$, etc.). En janvier 1871, en réponse à une lettre de Maxwell lui faisant part de son intention d'utiliser les quaternions, Price émettait quelques réserves sur ces objets mathématiques encore trop peu connus qui risquaient de "compromettre l'utilité " de l'ouvrage ${ }^{23}$. Price conseillait donc de n'écrire les formules sous forme de quaternions qu'en complément d'une présentation sous la forme, plus classique, de coordonnées cartésiennes. De fait, c'est effectivement ainsi que les quaternions furent utilisés dans le traité.

Après que Maxwell eut évalué la taille du livre à un millier de pages ${ }^{24}$, Price transmit la requête d'impression au Board of delegates qui décidait le 18 janvier 1871 d'imprimer l'ouvrage à mille cinq cents exemplaires ${ }^{25}$. L'impression du Treatise commença en février. Les premiers mois donnèrent lieu à quelques ajustements concernant les figures prévues dans l'ouvrage et le choix des symboles mathématiques employés ${ }^{26}$. Un différent survint en mars au sujet de la taille des lettres capitales employées pour le nom des points ${ }^{27}$. Maxwell obtint finalement gain de cause malgré le coût représenté par les modifications qu'il demandait. En mai 1871, les deux parties signaient le contrat les liant légalement ${ }^{28}$.

L'impression se poursuivit ensuite quasiment sans nouveaux échanges entre Maxwell et Price. Il est probable que, pendant cette phase, Maxwell communiquait directement avec l'imprimeur. Comme l'indique la corres-

18. Lettre du 19 février 1870, OUPA, Letters Book, vol. 3, p. 6-7.

19. Lettre du 4 janvier 1871, OUPA, Letters Book, vol. 4, p. 109-110. L'original de cette lettre est conservé à la Cambridge University Library, Add. mss 7656, p. 659. Harman en cite quelques extraits in MAxweu, 1995, p. 30, n. 90, p. 31, n. 95 et p. 636, n. 3.

20. Lettre du 3 mai 1870, OUPA, Letters Book, vol. 3, p. 112.

21. Lettre du 3 mai 1870, OUPA, Letters Book, vol. 3, p. 112.

22. Lettre du 21 septembre 1871, OUPA, Letters Book, vol. 3, p. 350.

23. Lettre du 4 janvier 1871, OUPA, Letters Book, vol. 4, p. 109-110.

24. Lettre du 7 janvier 1871, OUPA, Letters Book, vol. 4, p. 118.

25. OUPA, Order Book, 18 janvier 1871, et lettre du 18 janvier 1871, OUPA, Letters Book, vol. 4, p. 131

26. Lettre du 15 mars 1871, OUPA, Letters Book, vol. 4, p. 274.

27. Lettre du 24 mars 1871 et lettre du 11 avril 1871, OUPA, Letters Book, vol. 4, p. 301 et 346. Voir, aussi, in MaxweL, 1995, $\mathrm{n}^{\circ}$ 458, p. 856, la lettre de Maxwell du 26 mai 1873 relatant cette péripétie à William Strutt (lord Rayleigh).

28. Pour les envois du brouillon puis de la version définitive du contrat, voir lettre du 19 avril 1871, OUPA, Letters Book, vol. 4, p. 360 et lettre du 12 mai 1871, OUPA, Letters Book, vol. 4, p. 410. Un exemplaire du contrat est conservé à la Cambridge University Library, Add. mss 8812/159. 
pondance de Maxwell, les épreuves du Treatise étaient transmises à Tait et à Thomson pour commentaires ${ }^{29}$. Maxwell effectua ce travail pour le Reprint of papers on electrostatics and magnetism que Thomson publia en 1872. Pendant ce temps, Maxwell procédait aux dernières modifications de son texte. Toutefois, en juillet 1872, Maxwell écrivait à Price pour se plaindre de la lenteur de l'impression ${ }^{30}$. En décembre, il s'impatientait au point de suggérer que les deux volumes soient publiés séparément, l'impression du premier volume étant quasiment achevée. Cette requête se heurtait à un refus déterminé de Price qui mettait en avant les fortes « raisons commerciales " s'y opposant ${ }^{31}$. Enfin, le Treatise fut publié en mars 1873.

Outre le fait de dater plus précisément les différentes étapes de la production du Treatise, ce récit du processus de la publication nous a permis d'entrevoir comment les échanges de Maxwell avec différents interlocuteurs participaient à l'élaboration de son ouvrage. Ainsi, la réalisation du projet de Maxwell avait été favorisée par ses relations avec Tait et Thomson aussi bien sur le plan intellectuel que sur le plan institutionnel. De plus, nous verrons plus loin que le traité de Maxwell entretenait probablement des relations de complémentarité avec d'autres livres préparés par ses interlocuteurs : le Treatise on natural philosophy et le Reprint of papers on electrostatics and magnetism de Thomson. Si les échanges avec Price ne semblent porter que sur des aspects périphériques du livre touchant sa forme (taille de la page, symboles mathématiques, etc.) ou sa distribution (le prix de vente ou le nombre d'exemplaires imprimés), ils nous rappellent cependant que le projet de Maxwell devait s'intégrer à la politique éditoriale de la Clarendon Press. En particulier, ainsi que le laissait entendre Price à propos de l'usage des quaternions, le Treatise devait être suffisamment pédagogique pour justifier son appartenance à la «Clarendon Press Series». Ces exigences furent sans aucun doute respectées par Maxwell au cours de sa rédaction sans aucun échange épistolaire durant cette phase de la préparation du livre.

\section{II. - UN « SENATE-HOUSE TREATISE 》 : LE TREATISE ET LA RÉFORME DE L'ENSEIGNEMENT DE LA PHYSIQUE À CAMBRIDGE}

Entre 1866 et 1873, l'université de Cambridge a connu une série de réformes touchant principalement l'enseignement de la physique. Si cer-

29. Maxwel, 1995. Des épreuves du Treatise, annotées par Tait, sont conservées à la Cambridge University Library, Add. mss 7655, IV/2.

30. Lettre du 12 juillet 1872, OUPA, Letters Book, vol. 6, p. 213-214.

31. Lettre du 9 décembre 1872, OUPA, Letters Book, vol. 6, p. 460. 
taines études historiques ont déjà signalé l'importance du rôle de Maxwell dans ce processus ${ }^{32}$, elles ont en revanche moins prêté attention au fait que cette période coïncide avec la préparation du Treatise entre la fin de 1867 et le début de 1873. Il s'agit ici de chercher à mettre en lumière les relations entre ces deux aspects de l'activité de Maxwell au cours de cette période, généralement étudiés séparément. D'une part, la décision d'une réforme du Mathematical Tripos à Cambridge a sans doute fortement incité Maxwell à concevoir le projet d'un traité sur l'électricité et le magnétisme. D'autre part, la mise en ouvre efficace de cette réforme dépendait en grande partie de la publication d'un tel ouvrage. Il faut, enfin, replacer les réformes à Cambridge et la publication du Treatise dans un contexte plus général qui favorisa vers 1865 l'introduction, dans de nombreuses universités britanniques, de laboratoires de physique destinés à enseigner les pratiques de mesure de précision employées principalement pour l'étude de la chaleur, de l'électricité et du magnétisme.

Depuis le début du siècle, des étudiants venaient à Cambridge de tout le Royaume-Uni pour passer le prestigieux examen du Mathematical Tripos. Délivrant le titre de bachelor of arts avec les mathematical honours, l'examen portait sur des sujets de mathématiques ou de physique mathématique et intervenait après plus de trois années de préparation dans les collèges. Les étudiants classés parmi les quarante premiers (sur une centaine de candidats environ) étaient désignés par le titre de wrangler. Une telle place donnait accès à une brillante carrière ecclésiastique, juridique ou politique et, parfois, pour les mieux classés désirant se lancer dans la recherche en physique ou en mathématiques, à une carrière d'enseignant à l'université. Le prestige de l'examen était tel qu'au milieu du siècle, une grande partie des professeurs de mathématiques ou de philosophie naturelle des universités britanniques étaient d'anciens wranglers.

En 1848, une réforme avait abouti à la création d'un Board of mathematical studies comprenant, entre autres, les six professeurs de mathématiques et de physique de Cambridge. Cette commission était chargée de nommer les examinateurs, de déterminer le contenu des programmes et de publier un rapport annuel sur l'examen. Dans ses deux premiers rapports, le Board of mathematical studies excluait des sujets sur lesquels «les recherches [étaient] encore imparfaites, insatisfaisantes ou pour lesquels les mathématiciens ne s'accord[ai]ent pas encore suffisamment ". Ces critères justifièrent l'exclusion de questions sur la chaleur, l'électricité ou le magnétisme ${ }^{33}$.

32. Voir, en part., Sviedrys, 1970, et WILSON, 1982. Sviedrys soutient, p. 140, que le souci de réformer l'enseignement à Cambridge a motivé la démission de Maxwell à King's College en 1865. Cette thèse est séduisante mais n'est, à ma connaissance, pas documentée.

33. WILSON, 1982. 
Toutefois, au cours des années 1860 , plusieurs voix s'élevèrent pour regretter que le Mathematical Tripos fasse une place insignifiante aux sujets sur lesquels portaient alors des recherches importantes en physique. En particulier, l'astronome royal, George Biddell Airy, lui-même ancien wrangler, émit publiquement de telles critiques sur le Mathematical Tripos en 1864 lors d'une conférence prononcée à Cambridge. Le 8 mai 1867, il donnait un tour plus officiel à ses recommandations en adressant une lettre au vice-chancellor de l'université. Il y précisait les sujets qui lui semblaient devoir être abordés dans le cadre du Mathematical Tripos et détaillait les difficultés à surmonter dans cette entreprise. Airy désignait comme principales raisons à la prévisible lenteur d'une telle réforme le manque de manuels adaptés aux besoins de l'examen et la difficulté à former des examinateurs compétents. Il remarquait, en particulier, que la production d'ouvrages adéquats demandait un travail important pour une rémunération négligeable ${ }^{34}$.

La lettre de l'astronome royal fut transmise au Board of mathematical studies et donna lieu à une série de discussions en son sein. Il semble que l'illustre physicien et professeur de mathématiques à Cambridge, George Gabriel Stokes, joua un rôle primordial dans l'élaboration d'un projet de réforme soumis le 8 mai 1867 dans un rapport au vice-chancellor. Cette réforme visait à permettre d'introduire de nouveaux sujets dans l'examen tout en évitant que les étudiants ne cherchent à maitriser une somme excessive de connaissances. Le rapport proposait donc de subdiviser les différents sujets en plusieurs grandes sections représentées par un nombre fixe de points, afin de permettre aux meilleurs étudiants de choisir leur domaine de prédilection. La création d'une section formée par les sujets sur la chaleur, l'électricité et le magnétisme constituait la principale innovation ${ }^{35}$. Le 2 juin 1868, cette réforme était approuvée par le Conseil de l'université qui décidait sa mise en ouvre pour la session de 1873.

Bien que ne résidant qu'occasionnellement à Cambridge, Maxwell joua un rôle actif dans le processus qui conduisit à cette réforme. Membre du Board of mathematical studies depuis novembre 1866, il fut un des signataires du rapport délivré en mai 1867. Mais son influence fut surtout perçue à travers son action comme examinateur du Mathematical Tripos en 1866 et 1867 . L'originalité de ses questions semble avoir beaucoup fait pour pro-

34. AYRY, 1896, p. 255-259: «The real difficulties, and they are not light ones, would probably be found in providing Examiners and Books. At present both are wanting within the University. Where there is a great and well-founded objection to instrusting examinations to persons foreign to the University, and where the books have to be created with labour and with absolute outlay of money (for their sale could never be remunerative), the progress must be slow. "

35. Ce rapport est inclus dans les Minutes of the Board of mathematical studies, Cambridge University Archives. 
mouvoir une plus grande ouverture de l'examen vers la recherche en physique. Un témoignage, cité dans la biographie de Maxwell, nous donne l'appréciation d'un contemporain sur son rôle dans la réforme :

"The style of mathematics which was popular in Cambridge for some time before was, to say the least, one-sided, and one-sided in a somewhat unproductive direction. There were many complaints that Cambridge was behind the rest of the scientific world, and that, whereas the students of so many other Universities were introduced to the splendid discoveries of such subjects as Electricity and Heat, the Wranglers of Cambridge spent their time upon mathematical trifles and problems, so-called, barren alike of practical results and scientific interest. Maxwell's questions (as Moderator in 1866) infused fresh life into the Cambridge Tripos, and, therefore, into the University studies, by the number of original ideas and new lines of thought opened up by them, thus preparing for the change of system in 1873, when so many interesting subjects were added to the Examination ${ }^{36}$."

Maxwell fut également mis à contribution entre 1868 et 1873 pour préparer la mise en place de la réforme. Fait rare, il fut à nouveau nommé examinateur en 1869 et 1870 , sans doute pour assurer une transition progressive vers le nouveau système. Mais, surtout, il rédigea au cours de cette période deux ouvrages, le Theory of heat (1871) et le Treatise on electricity and magnetism (1873) qui couvraient à eux deux les sujets introduits dans l'examen. Le Treatise, exigeant un niveau mathématique élevé, était particulièrement adapté au Mathematical Tripos. Maxwell annonça son projet d'écrire un traité sur l'électricité et le magnétisme en novembre 1867, soit seulement six mois après le rapport proposant d'élargir l'examen aux « nouveaux sujets ». Dès cette date, Tait faisait allusion aux rapports entre l'examen de Cambridge et le projet de Maxwell en qualifiant son livre de "Senate-House Treatise ${ }^{37}$ ". Examinateur conforme aux souhaits de l'astronome royal, Maxwell se disposait donc aussi à produire le type d'ouvrage dont Airy déplorait l'absence. Il était, en effet, l'oiseau rare qui disposait du temps, de la compétence et de l'aisance financière lui permettant de réaliser le travail nécessaire à cette entreprise sans en attendre une rémunération significative.

La réforme du Mathematical Tripos conduisit à d'autres nouveautés à Cambridge. Le 27 novembre 1869, le Conseil de l'université décidait de créer un Physical Science Promotion Syndicate chargé de trouver les moyens d'enseigner à Cambridge l'étude expérimentale de la chaleur, de l'électricité et du magnétisme. Le 27 février 1869, le Syndicate publiait un

36. Campbell \& Garnett, 1882, p. 356-357.

37. Le Mathematical Tripos était désigné à Cambridge sous le terme de «Senate-House Examination" d'après le nom du bâtiment où les résultats étaient proclamés. 
rapport dans lequel il préconisait la création d'une nouvelle chaire de physique expérimentale accompagnée d'un poste de démonstrateur. Afin que le nouveau professeur puisse exercer son enseignement dans de bonnes conditions, le Syndicate recommandait également la construction d'un nouveau laboratoire, équipé des appareils nécessaires aux travaux expérimentaux. Ses recommandations ne furent pas immédiatement suivies d'effet, du fait des sommes importantes nécessaires à leur réalisation. Cependant, en octobre 1870 , le septième duc du Devonshire, chancellor de l'université, proposait de délivrer les fonds nécessaires à la construction du laboratoire et à l'acquisition des appareils expérimentaux. Cette offre princière permettait alors de débloquer rapidement la situation. Le 28 novembre, le Conseil de l'université proposait la création de la nouvelle chaire de physique expérimentale et le 9 février 1871 la décision était entérinée. L'université fit également le nécessaire pour lancer la construction du laboratoire. Et le $1^{\text {er }}$ mai 1874 était inauguré le bâtiment qui allait abriter le fameux Cavendish Laboratory ${ }^{38}$.

Après le refus de William Thomson, puis de Hermann von Helmholtz, d'être candidat à la nouvelle chaire ${ }^{39}$, Maxwell se vit sollicité en février 1871 par plusieurs membres de l'université dont le très influent professeur Stokes ${ }^{40}$. Quelques jours plus tard, il décidait de se porter candidat et le 8 mars il était élu sans opposition. Le prestige de Maxwell ne rivalisait pas, à cette époque, avec celui de Thomson ou de Helmholtz. Son élection était donc probablement due aussi bien à son rôle prépondérant dans la promotion de l'enseignement de l'électricité, du magnétisme et de la chaleur à Cambridge qu'à ses articles sur l'électromagnétisme et la théorie cinétique des gaz.

Le Treatise on electricity and magnetism, dont l'impression commençait, était alors attendu à Cambridge par les étudiants préparant la nouvelle formule du Mathematical Tripos. Malheureusement, malgré deux lettres de Maxwell à la Clarendon Press pour protester contre la lenteur de l'impression et pour suggérer une édition anticipée du premier volume ${ }^{41}$, l'ouvrage ne parut qu'en mars 1873, soit deux mois après la session du Mathematical Tripos qui inaugurait la nouvelle formule. Pour évaluer l'importance du Treatise dans l'introduction de questions sur l'électricité dans le Mathematical Tripos, il est particulièrement instructif de se pencher sur cette première année de mise en place du nouveau système. Maxwell avait été nommé additional examiner afin d'assister les quatre examinateurs dans l'introduction des « nouveaux sujets » de physique. Le Cambridge Univer-

38. SCHUSTER, 1910, p. 1-13.

39. ThOMPSON, 1910 , p. 557-566.

40. MAXWELL, 1995, no 357, p. 611-613.

41. Voir première partie de cet article, p. 519. 
sity Reporter du 20 mai 1873 contient un compte rendu d'une réunion, le 15 mai 1873, des principaux protagonistes de la réforme à l'occasion du rapport du Board of mathematical studies sur le déroulement de l'examen. Le plus prestigieux private coach de l'époque, Edward J. Routh s'y étonnait du peu de questions posées sur l'électricité :

"Electricity, a long subject, with hardly any book to be read, had only two [questions], though Sir William Thomson's book extended to 600 pages, and Professor Clerk-Maxwell's to two thick volumes. "

Maxwell et un autre examinateur, Norman $M$. Ferrers, expliquèrent que la décision de ne poser que très peu de questions en électricité était due au fait que les livres de Maxwell et de Thomson n'étaient pas encore parus au moment de l'examen :

« [Mr Ferrers said that] With respect to Electricity, Professor Maxwell's book did not exist at the time when the candidates were preparing for the examination, and Sir William Thomson's Essays had not been published in a collected form.

[...] Professor Clerk-Maxwell allowed that some subjects had been disproportionately represented. Mr Routh had compared subjects in which books existed with others in which there appeared to be no books to be read. On account of this difficulty respecting books, and on account of the newness of the subjects, Electricity and Magnetism had not received full attention in the examination. "

Les principaux acteurs de la réforme, parmi lesquels Maxwell lui-même, établissaient donc un lien explicite entre la possibilité d'interroger les candidats sur l'électricité et la parution du Treatise on electricity and magnetism. Cet ouvrage, ainsi que le Reprint of papers on electrostatics and magnetism de Thomson, paru fin 1872, étaient les seuls jugés adaptés à la préparation des étudiants ${ }^{42}$.

L'année suivante, le Student's Guide for the university of Cambridge conseillait trois ouvrages sur l'électricité pour la préparation du Mathematical Tripos: le Treatise de Maxwell, le Reprint de Thomson et l'Electricity and magnetism de Fleeming Jenkin ${ }^{43}$. Nous verrons plus loin les particularités du Treatise par rapport à ces deux ouvrages. Le récit de Richard Tetley Glazebrook, wrangler en 1876, témoigne du rôle de l'ouvrage de Maxwell dans la préparation de l'examen par les meilleurs étudiants :

"My own life as an undergraduate began in October 1872, a few months before the first examination (January 1873) under the new schedule, including Heat, Electricity, and Magnetism. [...]

42. Voir aussi WiLson, 1982 , p. 338.

43. Student's Guide for the university of Cambridge, 1874, p. 131. 
It was a time of great interest; there were few or no text-books, Fleeming Jenkins' Electricity and magnetism in Longmans' Text-book of Science Series appeared in 1873, Maxwell's Heat in the same series was published about the same time as well as his Electricity and magnetism. The subjects were new to our teachers as well as to ourselves. Thomas Dale of Trinity was my coach. [...] My copy of Maxwell's Electricity was bought, I think, during the Long Vacation of 1874. I have it still, a few Chapters or Sections of Volume I marked with Dale's $R$, others that we should now think most interesting with an $O$. Volume II I hardly touched until after the Tripos when, in the summer of 1876, I went with Dale to Buttermere and we read much of it together ${ }^{44}$."

La centaine d'étudiants qui préparaient le Mathematical Tripos chaque année constituait donc une partie importante des acheteurs potentiels auxquels étaient destinés les mille cinq cents exemplaires du Treatise. Il est probable qu'ils contribuèrent de façon significative aux trois cent quatrevingt-huit ventes effectuées entre mars et juin 1873 et à la centaine de ventes annuelles durant la décennie suivante ${ }^{45}$.

Mais les réformes à Cambridge doivent aussi être situées dans un contexte plus général. Graeme Gooday a analysé de façon convaincante l'émergence vers 1865 de laboratoires de physique à vocation pédagogique dans les universités britanniques. Il l'explique, d'une part, par le développement des techniques de mesure de précision dans des disciplines telles que la thermodynamique et l'électromagnétisme et, d'autre part, par la reconnaissance institutionnelle des besoins d'un enseignement de ces techniques à l'université pour la formation des ingénieurs et des enseignants ${ }^{46}$.

Dans cette évolution, Thomson fait figure de pionnier. Dès le début de sa carrière, ses travaux sur l'électromagnétisme et la théorie de la chaleur ont exercé une influence considérable. Sur le plan théorique, il fut un des principaux artisans des principes énergétiques qui jouèrent un rôle central dans les recherches menées au cours des décennies suivantes ${ }^{47}$. D'autre part, il développa à partir de 1846 des recherches expérimentales qui lui permirent de développer des techniques et des instruments de mesure de précision. Ces travaux, menés à l'université de Glasgow avec l'aide de quelques-uns de ses étudiants, aboutirent en 1858 à la création officielle du premier laboratoire de physique au sein d'une université britannique. De plus, sa participation, entre 1856 et 1866 , à l'installation d'un câble télégraphique sousmarin entre l'Angleterre et les États-Unis favorisa fortement la promotion

44. Sir Richard Tetley Glazerrook, " Early days at the Cavendish Laboratory », in ThomSON, 1931, p. 131-133.

45. OupA, Ledger Book, p. 34, 179.

46. GOODAY, 1990.

47. MOYER, 1977; MOYer, 1978; SMITH, 1978; WiSE, 1979. 
dans l'industrie télégraphique des mesures scientifiques de précision au détriment des méthodes empiriques par «essais et erreurs " employées jusqu'alors. Le rôle essentiel de ses méthodes et de ses instruments de mesure dans le mémorable succès de cette entreprise en 1866 lui valut d'être fait chevalier la même année ${ }^{48}$.

En 1861, la British Association for the advancement of science créait, à l'initiative de Thomson, un comité chargé de définir une norme de résistance électrique afin de satisfaire les besoins naissants de l'industrie télégraphique et de la recherche en électromagnétisme. Les normes définies par le comité faisaient appel à des principes théoriques (système d'unités absolues de Wilhelm Eduard Weber et principe de conservation de l'énergie) que Thomson avait déjà utilisés pour construire ses propres instruments. Les normes et les instruments développés par la suite pour l'industrie, la recherche et l'enseignement matérialisaient ainsi les résultats théoriques et pratiques des travaux menés depuis près de vingt ans sur l'électromagnétisme et la théorie de la chaleur ${ }^{49}$. Parmi les membres les plus actifs de ce comité figuraient, d'ailleurs, deux futurs auteurs d'ouvrage sur l'électricité et le magnétisme, Jenkin et Maxwell.

Grâce à ces succès scientifiques et industriels, les travaux sur l'électricité, le magnétisme et la chaleur furent intégrés dans l'enseignement délivré par les universités britanniques. À partir de 1865 , plusieurs d'entre elles créaient des chaires pour enseigner les pratiques expérimentales qui leur étaient associées ${ }^{50}$. Ces innovations allaient de pair avec la création de laboratoires de physique destinés à la formation des étudiants dans ces institutions comme dans d'autres lieux universitaires possédant déjà une chaire de philosophie naturelle ${ }^{51}$. Les réformes à Cambridge s'inscrivaient également dans ce mouvement de reconnaissance des recherches sur la chaleur et l'électromagnétisme. L'introduction de ces disciplines dans le Mathematical Tripos manifestait le fait que les recherches étaient devenues, aux yeux des membres du Board of mathematical studies, moins « insatisfaisantes » sur le plan théorique et mathématique qu'en $1848^{52}$. De plus, la création de la chaire de physique expérimentale et du Cavendish Laboratory prenait en compte le fait que ces progrès théoriques étaient indissociables des pratiques de mesure de précision qui les avaient accompagnés.

48. SMITH \& WISE, 1989, chap. XIX et XX.

49. GOODAY, 1990 , p. 34-35.

50. Par ex., chaire de philosophie expérimentale à Oxford (1865), chaire de physique au Royal College of Mines (1869), subdivision de la chaire de philosophie naturelle en deux chaires de physique mathématique et physique expérimentale à University College London (1865) et Owens College Manchester (1870).

51. Université de Glasgow (1866), université d'Édimbourg (1868) et King's College à Londres (1868).

52. Winson, 1982, p. 338. 
Les réformes à Cambridge furent probablement également favorisées par une vaste campagne en faveur de l'enseignement des sciences menée à l'occasion de l'exposition universelle de Paris en 1867. Tirant argument d'une spectaculaire diminution du nombre des prix accordés aux participants britanniques à l'exposition, de nombreux observateurs dénoncèrent le déclin de l'industrie britannique face à ses concurrents continentaux. Cet échec était attribué aux méthodes empiriques des patrons britanniques jugées inefficaces face à celles de leurs homologues d'Europe continentale supposés pétris de culture scientifique. L'émoi fut tel qu'on forma une commission gouvernementale en 1869 pour enquêter sur l'état de l'instruction scientifique dans le Royaume-Uni : la Royal Commission on scientific instruction and the advancement of science bientôt appelée Devonshire Commission du nom de son président, le septième duc du Devonshire ${ }^{53}$. Ce dernier, chancellor de l'université de Cambridge, fut celui-là même qui annonça en octobre 1870 son intention de financer sur ses propres deniers la construction du Cavendish Laboratory et qui permit ainsi la création de la chaire de physique expérimentale qu'allait occuper Maxwell. Ce geste manifestait son désir de voir se développer, dans son université, l'enseignement de connaissances qu'il jugeait utiles à l'industrie. En effet, diplômé du Mathematical Tripos, il était également un industriel de la métallurgie attentif aux applications de la chimie et de la physique dans ce domaine ${ }^{54}$.

La publication du traité de Maxwell s'inscrivait directement dans ce processus. Ainsi, dans la préface de son ouvrage, Maxwell rappelait le rôle important de l'industrie télégraphique dans le développement de l'électromagnétisme et particulièrement pour la mesure des phénomènes :

"The important applications of electromagnetism to telegraphy have also reacted on pure science by giving a commercial value to accurate electrical measurements, and by affording to electricians the use of apparatus on a scale which greatly transcends that of any ordinary laboratory. The consequences of this demand for electrical knowledge, and of these experimental opportunities for acquiring it, have been already very great, both in stimulating the energies of advanced electricians, and in diffusing among practical men a degree of accurate knowledge which is likely to conduce to the general scientific progress of the whole engineering profession ${ }^{55}$."

Il faisait en conséquence une place importante dans son ouvrage aux méthodes, théoriques et instrumentales, développées pour mesurer les phénomènes à travers la théorie de la mesure de Gauss et Weber et des cha-

53. Sur les conséquences de l'exposition universelle de 1867 et la Devonshire Commission, voir CARDWELL, 1957, p. 84-97.

54. SvIEDRYs, 1970, p. 139-140.

55. MAXWELL, 1873, p. vil. 
pitres consacrés à la description des appareils de mesure. Le travail de définition de normes électriques qu'il avait entamé dans le cadre de sa participation au comité de la British Association for the advancement of science fut poursuivi à partir de 1874 au Cavendish Laboratory ${ }^{56}$.

III. - LE CONTEXTE ÉdTORIAL DE LA PUBlication DU TREATISE

Vers le début des années 1870 , le contexte universitaire et industriel était donc favorable à la parution d'ouvrages sur l'électricité et le magnétisme. Plus particulièrement, la réforme du Mathematical Tripos appelait la parution d'un livre adapté à cet examen. Le manque d'ouvrages était si nettement ressenti que, face à la parution trop tardive du Reprint of papers on electrostatics and magnetism de Thomson fin 1872 et du Treatise de Maxwell début 1873, les examinateurs de la session de 1873 inaugurant le nouveau système avaient dû renoncer à interroger les candidats sur l'électricité et le magnétisme. Un des rares ouvrages importants traitant en anglais de l'électricité et du magnétisme, le Traité sur l'électricité du physicien suisse Auguste de La Rive dont les trois volumes avaient été traduits et publiés en Grande-Bretagne entre 1853 et 1858 , n'était malheureusement pas adapté aux besoins du Mathematical Tripos. En effet, il ne proposait pas un traitement mathématique du sujet et n'intégrait pas certains développements théoriques récents - en particulier, les principes énergétiques - qui avaient fortement favorisé l'introduction des nouveaux sujets.

Cependant, le Treatise on natural philosophy de Thomson et Tait, dont le premier volume sur la dynamique était paru en octobre 1867, paraissait particulièrement bien placé pour combler les besoins créés par la réforme du Mathematical Tripos. Comme les auteurs l'indiquaient dans la préface, leur projet initial consistait à proposer en quatre volumes « un exposé raisonnablement complet de ce que l'on sait en philosophie naturelle ». Les professeurs de Glasgow et d'Édimbourg avaient spécialement prévu de traiter l'électricité et le magnétisme ${ }^{57}$. D'autre part, la nature de l'ouvrage était conforme aux besoins du Mathematical Tripos puisqu'il comportait, à côté d'un exposé «adapté au lecteur non mathématicien ", un traitement mathématique très complet et élaboré. De plus, l'ouvrage intégrait dès l'exposé des lois fondamentales de la dynamique, le principe de la conser-

56. SChafFer, 1992, et SCHAFFer, 1995.

57. Voir le récit de l'élaboration du Treatise on natural philosophy et les citations de la correspondance entre Tait et Thomson au sujet de leur ouvrage, in THомpson, 1910, p. 447480. 
vation de l'énergie dont Thomson était l'un des principaux artisans. L'ouvrage bénéficiait, enfin, du prestige considérable de Thomson qui, au cours des décennies précédentes, avait été à l'origine d'importantes transformations théoriques et institutionnelles de la physique britannique. Dès la sortie du premier volume, l'ouvrage bientôt appelé " $T \& T^{38}$ " avait suscité un accueil enthousiaste dans la communauté britannique, certains allant jusqu'à le comparer aux Principia de Newton et à la Mécanique céleste de Laplace.

On sait que les deux auteurs durent renoncer à exécuter la totalité de leur ambitieux projet. Ils ne purent qu'éditer une seconde édition largement augmentée du premier volume en deux tomes parus en 1879 et 1883 et annoncèrent, dans la préface de ce second tome, leur décision de s'en tenir là ${ }^{59}$. Il est probable que Maxwell ne put former son projet de traité sur l'électricité et le magnétisme en 1867 que parce que Thomson et Tait avaient dès cette date renoncé à traiter ce sujet dans leur ouvrage. Le public susceptible d'être intéressé par un traité mathématique de haut niveau sur l'électricité et le magnétisme était, semble-t-il, trop réduit pour que la Clarendon Press puisse envisager de publier deux ouvrages de ce type. Nous avons vu, en effet, que les ventes du Treatise s'élevèrent à une centaine d'exemplaires par an.

Une lettre du 23 octobre 1868 de Price à Tait confirme que le problème de la compatibilité des deux projets fut effectivement abordé par les protagonistes. Price s'y interrogeait sur l'opportunité d'accepter une offre d'un traité mathématique sur la thermodynamique sachant que Thomson et Tait envisageaient d'aborder ce sujet dans un prochain volume de leur Treatise on natural philosophy, concluant ainsi :

"[...] we might perhaps accept an offer of a good man for such work, as we have, with your cognizance, taken the offer of Clerk Maxwell for a book on Electricity \& Magnetism. Let me have your views \& intentions ${ }^{60}$."

Le 22 janvier 1869, suite à une réponse de Tait dont nous n'avons pas connaissance, Price l'informait que la Clarendon Press avait décidé de refuser l'ouvrage sur la thermodynamique ${ }^{61}$. Ces documents confirment donc que la Clarendon Press accepta le projet de Maxwell avec l'accord de Tait et de Thomson et que cet accord était interprété comme un renoncement à traiter le sujet dans leur propre ouvrage.

Il est aisé d'imaginer les raisons qui amenèrent Tait et Thomson à renoncer à exécuter l'intégralité de leur projet. En 1867, lorsque le premier

58. $T$ désignait Thomson et $T$ ', Tait, manifestant ainsi la différence de prestige entre les deux auteurs.

59. ThомPSon, 1910 , p. $474-480$.

60. Lettre du 23 octobre 1868, OUPA, Letters Book, vol. 1, p. 192-193.

61. Lettre du 22 janvier 1869, OUPA, Letters Book, vol. 1, p. 318. 
volume paraissait, les deux hommes travaillaient sur le projet depuis plus de cinq années. La lenteur de la rédaction était, semble-t-il, principalement le fait de Thomson, trop absorbé par ses nombreuses autres activités pour se consacrer pleinement à cet ouvrage. En juin 1864, Tait manifestait déjà son impatience envers les retards accumulés par son ami ${ }^{62}$. Dans ces conditions, il est probable que Tait et Thomson accueillirent avec soulagement le projet de Maxwell d'écrire un vaste traité sur l'électricité et le magnétisme qui les dispensait d'une partie de la tâche qu'ils s'étaient fixée. Le processus par lequel ils furent conduits à renoncer progressivement à la majeure partie de leur entreprise est rapporté par Silvanus Thompson dans sa biographie de William Thomson:

"I asked Lord Kelvin [William Thomson] once why no more than Vol. I had been given to the world. His reply was that the ground they had proposed to cover had in the years that followed been largely covered by such books as Rayleigh's Sound (1873) [sic], Maxwell's Electricity and magnetism (1873), and Lamb's Hydrodynamics (1879), and that he and Tait had so much specialized work of their own that they felt they had better frankly abandon the idea of proceeding further ${ }^{63}$.»

Il semble donc que les protagonistes considéraient en partie le Treatise de Maxwell comme un prolongement du Treatise on natural philosophy. Sur le plan théorique, Maxwell utilisait les notions et les méthodes introduites dans le T \& T' pour développer son propre exposé. En particulier, le fait que Tait et Thomson donnait dans leur ouvrage une importance centrale à la notion d'énergie permettait à Maxwell d'utiliser fréquemment ce concept encore récent sans éprouver le besoin de le définir à nouveau ${ }^{64}$.

Il est également instructif de comparer les conditions de l'élaboration du Treatise de Maxwell avec celles qui ont présidé à la publication, fin 1872, du Reprint of papers on electrostatics and magnetism de Thomson. Comme le Treatise, la réimpression des articles de Thomson sur l'électrostatique et le magnétisme fut très certainement favorisée par la réforme du Mathemati-

62. Voir in Thомpson, 1910, p. 467, lettre de Tait à Thomson: "I wish you would go ahead. I am getting quite sick of the Great Book, for I see plainly that if you don't send immediately the whole mass of your self-imposed contribution to Elastic Bodies we shall not get out in September. "

63. ThомpSon, 1910, p. 476-477. L'ouvrage de Rayleigh, Theory of sound, ne fut envisagé qu'en 1871 et le premier volume ne fut publié qu'en 1877 : voir R. Bruce LiNDSAY, «Strutt, John William, third baron Rayleigh ${ }^{2}, D S B$, vol. 13, p. 100-107. C'est donc probablement le projet de Maxwell qui, dès 1867, incita Thomson et Tait à restreindre leur entreprise.

64. On trouve une illustration du lien que Maxwell établissait entre le T \& T' et son propre ouvrage dans une lettre du 20 février 1868 adressée à Thomson, voir MAXwELl, 1995, $\mathrm{n}^{\circ}$ 281, p. 347: "I am glad to see a good deal of Spherical Harmonics in T.T' which relieve me" (c'est-à-dire : me dispense de traiter ce sujet dans mon propre livre). 
cal Tripos. Nous avons vu que, comme le traité de Maxwell, cet ouvrage était attendu à Cambridge pour permettre aux étudiants de préparer l'examen. De plus, les archives de l'Oxford University Press nous apprennent que Thomson conçut ce projet autour de juillet 1867 , soit quelques semaines seulement après que le Board of mathematical studies eut annoncé une réforme de l'examen. En effet, la Clarendon Press déclina à cette date une proposition de Thomson d'éditer un recueil de ses articles ${ }^{65}$.

Le refus de la Clarendon Press doit probablement être attribué au fait qu'un recueil d'articles ne constituait pas un ouvrage suffisamment pédagogique pour s'intégrer à sa politique éditoriale. Chaque article avait été publié dans des revues scientifiques de haut niveau et s'adressait à des spécialistes de la discipline. La juxtaposition des différents textes de Thomson, même s'ils abordaient presque tous les aspects de l'électrostatique et du magnétisme, ne constituait pas un exposé accessible aux profanes. Il est probable qu'un tel projet n'eût d'ailleurs pas vu le jour si Thomson et Tait avaient eu l'intention de faire paraître rapidement, dans leur Treatise on natural philosophy, un exposé systématique des connaissances du moment sur ces sujets. Le recueil d'articles de Thomson constituait donc probablement une solution de substitution destinée à pallier son manque de disponibilité pour un ouvrage de plus grande ampleur.

Quelques mois après avoir décliné la proposition de Thomson, la Clarendon Press acceptait le projet de Maxwell. Le recueil de Thomson était quant à lui publié chez l'éditeur londonien Macmillan, lui-même collaborateur de la Clarendon Press. Les relations entre les deux livres n'étaient sans doute pas dénuées d'ambiguiité. Pour une part, le Reprint de Thomson pouvait apparaître comme complémentaire du Treatise de Maxwell. Il donnait accès à certains textes de Thomson auxquels Maxwell faisait référence dans son propre exposé et, de façon plus générale, permettait d'étudier directement les articles qui avaient joué un rôle prépondérant dans une évolution théorique dont le Treatise pouvait représenter un aboutissement.

Mais les deux ouvrages se trouvaient également en position de concurrence auprès des étudiants du Mathematical Tripos dont certains allaient constituer une nouvelle génération de physiciens ${ }^{66}$. Le Reprint avait aussi pour fonction de manifester la contribution scientifique de Thomson

65. OUPA, registre du School Book Committee : «Thomson's papers declined. » Par ailleurs, Maxwell fait explicitement référence à ce projet dans une lettre adressée à Thomson et datée du 12 mai 1869, voir MAXweL, 1995, $n^{\circ} 321$, p. 482 : «How is the reprint of your electrical papers getting on? "

66. Le sentiment de compétition entre Maxwell et Thomson, à travers leur ouvrage respectif, est exprimé par ce demier dans une lettre à Tait du 21 août 1871, voir MAXWEL, 1995, $\mathrm{n}^{\circ} 383$, p. 675, n. 1 (c'est moi qui souligne) : «Dr T'I have at last got to the reprint of electrical papers, and in doing Math Th. of Magnetism \$\$ 8-13 have been forced to bring to an issue the long impeding question what is the magnetic axis of a magnet and what is its proper centre. Maxwell is (?) sure (?) to have it in his book so this is a race and you as Sec RSE 
comme distincte et antérieure à celle de Maxwell. D'une part, Thomson avait été attentif à marquer sa priorité dans l'élaboration de certaines méthodes en reproduisant ses textes sans modification et en précisant systématiquement leur date de publication; le recueil intégrait même la reproduction d'anciens textes manuscrits non publiés jusqu'alors pour lesquels était soigneusement précisée la date de rédaction ${ }^{67}$. D'autre part, Thomson s'était efforcé d'atténuer le caractère fragmentaire de son ouvrage, peu propice à attirer un public désireux d'apprendre l'électromagnétisme, en préférant un regroupement thématique des articles plutôt qu'une suite chronologique. Il avait également tenté de combler les lacunes de son traitement en ajoutant des textes nouveaux et en associant aux textes anciens des notes indiquant des méthodes ou des termes introduits plus récemment. Cependant, malgré ces ajustements destinés à adapter le livre à un contexte pédagogique et malgré le prestige de Thomson, le Reprint demeurait, par sa nature même, moins bien placé que le Treatise de Maxwell pour constituer l'ouvrage de référence de jeunes hommes désireux de se former à l'étude mathématique de l'électromagnétisme.

La période était également favorable à la parution d'ouvrages plus élémentaires sur l'électricité et le magnétisme qui n'entraient pas en concurrence avec le Treatise. Ainsi, en février 1868, peu après avoir annoncé son projet à Thomson et Tait et avant de l'avoir soumis à la Clarendon Press, Maxwell apprenait que cet organisme avait déjà prévu de publier un autre livre sur l'électricité. Sa réaction, exprimée dans une lettre adressée à Thomson, est significative de la façon dont il concevait la compatibilité des projets :

"I see by advertisements that Mr Esson of Merton Coll Oxford is going to do an Electricity for the Clarendon Press. I only know Mr Esson as an observer of the progress of chemical changes along with Mr Vernon Harcourt, so I do not know whether his book will be about Green's Theorem or about the influence of electrical action on plants. If the latter I shall go on with the theorem ${ }^{68}$.

Ainsi, le projet de Maxwell ne pouvait, à ses yeux, se trouver remis en cause que si le livre de William Esson proposait un traitement mathéma-

[Secretary of the Royal Society of Edinburgh] are bound to see fair play. " Voir aussi Peter M. HaRman, introd., in ibid., p. 25.

67. Par ex., Thomson, 1872, art. 524, note du chap. v1: " [Note, October 1871. - This chapter was written twenty-two years ago, and has lain in manuscript ever since, because I had not succeeded in finding time to write a sequel on inverse problems. It is now printed from the original manuscript with only a few verbal alterations, and it will be followed in this volume (chap. $x$ ) by the long-projected article on inverse problems, of which something was communicated to the British Association at its Oxford Meeting of 1847, but not published except in the very short abstract contained in the Report of that meeting.] "

68. MaXWelL, 1995, no 281, p. 346. 
tique de haut niveau, symbolisé ici par le théorème de Green. Cette éventualité lui semblait peu probable car les contributions scientifiques d'Esson en électromagnétisme lui étaient inconnues. De fait, le prestige scientifique d'Esson n'était pas comparable à celui de Maxwell. De huit ans plus jeune que ce dernier, il avait fait des études mathématiques à Oxford et avait été nommé à un poste d'enseignant à Merton College. Ainsi que le signalait Maxwell, ses principales contributions scientifiques portaient sur la chimie, domaine dans lequel il avait publié deux articles remarqués avec Vernon Harcourt, le professeur de chimie de l'université d'Oxford ${ }^{69}$. Dans ces conditions, il était plus vraisemblable que le livre d'Esson consiste en une description non mathématique des phénomènes électriques que Maxwell représentait, avec un humour un peu condescendant, par l'étude de l'action électrique sur les plantes. Si tel était le cas, Maxwell pouvait poursuivre la préparation de son propre livre.

Ces prévisions se trouvèrent vite confirmées. La Clarendon Press ne percevait aucune incompatibilité entre les deux publications puisque, lors de la parution du Treatise en mars 1873, le catalogue des ouvrages de la Clarendon Press annonçait encore la parution prochaine d'un Electricity par Esson. La différence entre les deux ouvrages était manifestée par la classification dont ils étaient l'objet : alors que le livre de Maxwell apparaissait sous la rubrique Mathematics, celui d'Esson était classé sous le titre Physical Sciences. Cette distinction correspondait à la séparation des études à Cambridge et à Oxford entre Mathematics et Natural Sciences ${ }^{70}$. L'examen associé aux Natural Sciences portait essentiellement sur les aspects expérimentaux des sciences étudiées et ne demandait qu'une connaissance élémentaire des mathématiques. La différence de statut entre Maxwell et Esson reflétait donc la prééminence du Mathematical Tripos sur le Natural Sciences Tripos.

Le livre d'Esson ne fut, en fait, jamais réalisé. Il semble que la Clarendon Press ait renoncé à ce projet dès juillet 1872 car, à cette date, Price écrivait à Maxwell pour lui proposer d'écrire un autre livre plus élémentaire sur le sujet :

"I hope you see your way to prepare for this Press an Elementary work on Electricity and Magnetism! Such as although not without Mathematics, it does not require such profound mathematical requirement as in this large Treatise. There are many reasons why we should desire to have such a book: The large book must be the source of much that will be written on the subject, and can-

69. Szabadvary, 1973.

70. Pour le Natural Sciences Tripos et le Mathematical Tripos à Cambridge, voir WILson, 1982. Je dois à Robert Fox des informations sur les études à Oxford. 
not have a very large sale. The small book will command a large sale; and the two should go together ${ }^{71}$.

Ainsi, selon Price, le Treatise se trouvait dans la position d'un ouvrage de référence, capable d'influencer des exposés plus élémentaires sur le même sujet. Il était donc important pour la Clarendon Press de publier rapidement un ouvrage de ce type écrit par le même auteur. Un tel livre promettait en effet d'être fort rémunérateur car, accessible à un plus large public, il aurait de plus bénéficié de la légitimité que Maxwell aurait acquise par la publication de son grand traité. Il s'agissait ainsi de réitérer le processus qui avait conduit Thomson et Tait à extraire des portions de texte de leur Treatise on natural philosophy pour former un court traité sur la dynamique exempt de mathématiques : les Elements of natural philosophy $(1873)^{72}$. Mais la tâche se révéla moins aisée que ne le prévoyait Price. Malgré de fréquentes lettres de la part de ce dernier, Maxwell n'acheva jamais la tédaction de ce livre du fait de ses multiples obligations à Cambridge. En 1881, deux ans après la mort de Maxwell, les manuscrits rédigés à cette occasion furent cependant édités par William Garnett, l'assistant de Maxwell à Cambridge, dans un livre intitulé Elementary Treatise in electricity.

Pour finir, il est utile de préciser la nature d'un autre ouvrage sur l'électricité et le magnétisme publié en 1873 : l'Electricity and magnetism de Fleeming Jenkin. Ce dernier, né en 1833, était un ingénieur spécialisé dans l'installation des câbles télégraphiques sous-marins. C'est dans le cadre de cette activité qu'il avait fait connaissance en 1859 avec William Thomson avec lequel il avait noué une solide amitié. Entre 1862 et 1869, il avait participé, en collaboration avec Maxwell, au comité de la British Association for the advancement of science pour la définition d'une norme de résistance électrique. Les deux hommes apportaient dans ce projet des compétences complémentaires : l'un étant plus porté sur les spéculations théoriques et l'autre davantage concerné par les réalisations techniques. Fellow de la Royal Society of London depuis 1865 , Jenkin avait été nommé professor of engineering à l'université d'Édimbourg ${ }^{73}$.

Le livre de Jenkin, paru comme le Theory of heat de Maxwell chez l'éditeur londonien Longmans, se voulait un traité adapté aux besoins d'électriciens confrontés à des applications pratiques. Le livre mettait particulièrement l'accent sur l'application des mesures et des lois électriques et

71. Lettre du 12 juillet 1972 (c'est moi qui souligne). Oupa, Letters Book, vol. 6, p. 213 214.

72. КнOTT, 1911, p. $201-202$.

73. Chipman, 1976. 
magnétiques à la télégraphie. Bien qu'orienté vers la mesure quantitative des phénomènes, le livre de Jenkin, s'adressant à des électriciens peu formés aux techniques mathématiques les plus élaborées, ne comportait pas de traitement mathématique aussi avancé que le Traité de Maxwell. À Cambridge, il était donc recommandé aux étudiants du Natural Sciences Tripos.

Sur le plan théorique, il semble que l'ouvrage de Jenkin ait dû beaucoup à Thomson et à Maxwell qui avaient tous deux été mis à contribution pour la relecture des épreuves. Les annotations de Maxwell sur les épreuves de Jenkin et leurs répercussions sur la forme définitive du livre suggèrent que l'expérience acquise par Maxwell dans la rédaction de son propre ouvrage eut une influence importante sur l'exposé de Jenkin ${ }^{74}$. Ainsi, avant même sa parution, le Treatise on electricity and magnetism exerçait-il sa fonction d'ouvrage de référence pour des exposés plus élémentaires. D'une façon plus générale, le livre de Jenkin se réclamait d'une nouvelle science de l'électricité issue des usages pratiques de l'électricité, notamment dans l'industrie télégraphique, et à laquelle étaient associés les noms de Faraday, Thomson et Maxwell. Jenkin opposait cette science à celle des « manuels ordinaires », dont l'ouvrage de De La Rive était désigné comme le principal représentant.

\section{IV. - LE TREATISE : UNE ENTREPRISE SCIENTIFIQUE À REPLACER DANS SON CONTEXTE}

Les études historiques consacrées à l'œuvre de Maxwell en électromagnétisme n'ont jusqu'ici que très peu mis en relation leur analyse du Treatise on electricity and magnetism avec les conditions institutionnelles de sa publication. Bien que certains auteurs aient évoqué le rôle du Treatise dans l'enseignement de l'électricité et du magnétisme à Cambridge ${ }^{75}$, ils présentent ce fait comme indépendant des principales innovations théoriques de l'ouvrage. Les études tendent ainsi à dissocier la démarche intellectuelle de Maxwell des contextes universitaire et éditorial dans lesquels elle s'est développée. L'influence considérable du traité sur la physique du $\mathrm{xx}^{\mathrm{e}}$ siècle a favorisé cette tendance en rendant négligeables, aux yeux des lecteurs modernes, les circonstances historiques locales de sa production ${ }^{76}$.

74. Voir les annotations de Maxwell sur les épreuves du livre de Jenkin dans les papiers de Maxwell, à la Cambridge University Library, Add. mss $7655 / \mathrm{Vc} / 41$, et, en part., les remarques sur la notion de potentiel.

75. Par ex., Simpson, 1970, p. 251, et SiEgel, 1991, p. 158.

76. Pour une analyse des réécritures de l'histoire des équations de Maxwell selon les développements les plus récents de la recherche en physique, voir GaLIson, 1983. Galison limite prudemment son analyse à l'histoire «spontanée » édifiée par des physiciens en fonction de leur propre programme et réalisation scientifique. 
S'attachant généralement à décrire l'évolution des conceptions de Maxwell en électromagnétisme, les récits historiques analysent ses différentes publications en tant qu'étapes successives de l'élaboration de la théorie. Selon cette perspective, le Treatise apparaît comme l'étape finale de l'élaboration, contenant la théorie arrivée à maturité. Les historiens ont donc souvent tendu à isoler la «théorie de Maxwell » de l'exposé général proposé par l'ouvrage. L'attention s'est alors portée de façon presque exclusive sur quelques chapitres supposés contenir les principales innovations théoriques de Maxwell ${ }^{77}$. Ces choix présentent l'inconvénient de laisser croire à un cloisonnement entre ces chapitres, conçus comme les seules parties où se seraient joués les enjeux scientifiques du livre, et le reste de l'ouvrage qui contiendrait un exposé élémentaire et dénué d'originalité, uniquement destiné à des novices. En étudiant le Treatise de la même façon que les articles publiés précédemment par Maxwell dans des revues spécialisées, l'historiographie n'a ainsi pas rendu compte des relations entre la nature spécifique de l'ouvrage, et notamment sa fonction pédagogique, et l'entreprise scientifique qu'il mettait en œuvre.

Une autre démarche est proposée ici : elle consiste à montrer que le contexte institutionnel de la publication et la nature de l'ouvrage doivent être pris en compte, non seulement pour comprendre la diffusion et le succès de la théorie de Maxwell, mais aussi pour analyser le texte lui-même. On peut certes concevoir le traité de Maxwell comme une « machine de guerre $^{78}$ " destinée à permettre une plus large reconnaissance de sa théorie. En effet, l'article «A dynamical theory of the electromagnetic field » paru en février 1865, que Maxwell lui-même considérait comme un texte de grande importance ${ }^{79}$, n'avait, semble-t-il, suscité que de faibles réactions dans la communauté scientifique en Grande-Bretagne et a fortiori sur le continent $^{80}$. Seul Helmholtz faisait référence à la théorie de Maxwell dans

77. Les analyses ont principalement porté sur la partie IV consacrée à l'électromagnétisme, en part. les chap. IV à IX, qui conduisent par une démarche déductive aux fameuses « équations générales du champ électromagnétique » et qui comportent de nombreuses similitudes avec l'article de 1865 « A dynamical theory of the electromagnetic field», et le chap. xx, qui expose la théorie électromagnétique de la lumière. On trouve également quelques analyses sur l'électrostatique (partie I) particulièrement centrées sur les passages suivants : art. 59 à 62 et art. 111.

78. Je dois cette expression à Michel Atten.

79. L'enthousiasme de Maxwell à ce sujet est exprimé dans une lettre datée du 5 janvier 1865 à son cousin Charles Hope Clay, lui-même classé $6^{\mathrm{e}}$ Wrangler du Mathematical Tripos de 1864, voir Campiell \& Garnett, 1882, p. 341-342 ou Maxwell, 1995, n 240, p. 203 : «I have also a paper afloat, with an electromagnetic theory of light, which, till I am convinced to the contrary, I hold to be great guns."

80. Une étude des articles sur l'électricité et le magnétisme publiés dans les Philosophical Transactions entre 1865 et 1873 montre que quasiment aucun ne fait référence à l'article de Maxwell. Ceci serait probablement confirmé si on étendait l'étude à d'autres revues britanniques. 
un article célèbre de 1870 sur les « équations du mouvement de l'électricité pour des conducteurs au repos ${ }^{81}$ ".

L'opportunité d'écrire un traité sur l'électricité et le magnétisme adapté aux nouveaux besoins des étudiants du Mathematical Tripos avait sans aucun doute l'attrait de faciliter la diffusion des conceptions de Maxwell. Cambridge demeurait, en effet, le lieu de formation le plus prestigieux du Royaume-Uni pour un jeune homme désireux de faire une carrière dans l'enseignement et la recherche en physique. À travers ce rôle central dans la formation des physiciens les plus considérés du pays, l'ouvrage de Maxwell était favorablement placé pour exercer à moyen terme une influence importante sur les recherches menées en électromagnétisme. De façon plus générale, le traité de Maxwell occupait la place d'un livre de référence dans ce domaine, consolidant ainsi la légitimité scientifique de son auteur. Le fait que Maxwell fut engagé dans la préparation d'un traité sur l'électricité et le magnétisme n'était d'ailleurs sans doute pas étranger à sa nomination en mars 1871 à la chaire de physique expérimentale de Cambridge.

Mais la description du contexte de la publication du Treatise, et en particulier de la fonction pédagogique qui lui était attribuée, permet aussi, et surtout, de mieux comprendre la nature même de l'entreprise scientifique que Maxwell entendait ainsi réaliser. L'élaboration des conceptions de Maxwell n'était d'ailleurs pas achevée avec la parution de l'article de 1865. Comme l'explique Daniel Siegel dans son livre Innovations in Maxwell's electromagnetic theory ${ }^{82}$, Maxwell tentait de résoudre dans son traité des problèmes encore non résolus par ses publications précédentes. L'article " A dynamical theory of the electromagnetic field " visait à proposer une théorie des phénomènes électromagnétiques indépendante du mécanisme des vortex moléculaires dans le cadre duquel les principales innovations de Maxwell avaient été formées. Malgré la réussite générale de ce programme, des incohérences formelles et conceptuelles demeuraient. En particulier, Maxwell aboutissait à une incompatibilité de certaines équations générales due à la difficulté à faire coïncider son traitement formel avec une représentation physique des phénomènes privilégiant la notion de champ sur celle de charge électrique. Ce n'est que dans le Treatise qu'il résolvait enfin ces difficultés. Il parvenait alors à établir la cohérence des équations générales tout en maintenant la primauté du champ. Pour cela, Maxwell proposait, dans la partie I consacrée à l'électrostatique, une " réinterprétation radicale » de la notion de déplacement et de ses relations avec la charge électrique. Elle n'était plus, en effet, considérée comme un concept premier mais comme un phénomène dû à la discontinuité du déplacement.

81. Pour une analyse de l'article de Helmholtz, voir Darricol, 1992, p. 232-239.

82. SIEGEL, 1991, p. 145-152. 
À partir de là, l'ensemble de l'entreprise mise en cuure par Maxwell à travers son traité doit être reconsidérée. L'introduction d'une représentation physique radicalement nouvelle d'un concept aussi fondamental que la notion de charge électrique représentait beaucoup plus qu'une innovation supplémentaire dans la théorie de Maxwell. Elle manifestait le projet d'une transformation complète de l'électromagnétisme. C'est cette entreprise que Maxwell présentait dans la préface du Treatise. Il y décrivait longuement, sur plus de trois pages, sa recherche comme une tentative d'expression mathématique des idées et des méthodes de Michael Faraday, afin de constituer une théorie des phénomènes électriques et magnétiques fondée sur la notion de champ électromagnétique. Cette théorie était présentée comme une alternative aux théories développées par des physiciens allemands tels que Wilhelm Weber ou Franz Neumann et fondées sur la notion d'action à distance. Le Treatise apparaissait alors comme un aboutissement de ce programme :

"These physical hypotheses [founded on the theory of action at a distance], however, are entirely alien from the way of looking at things which I adopt, and one object which I have in view is that some of those who wish to study electricity may, by reading this treatise, come to see that there is another way of treating the subject, which is no less fitted to explain the phenomena, and which, though in some parts it may appear less definite, corresponds, as I think, more faithfully with our actual knowledge, both in what it affirms and in what it leaves undecided ${ }^{83}$."

Il s'agissait donc d'enseigner une façon fondamentalement nouvelle de concevoir les phénomènes électromagnétiques. Un tel programme était d'une extrême ambition bien que Maxwell ait pris soin d'en atténuer l'audace en le plaçant sous l'égide du prestigieux nom de Faraday. Même si les conceptions du savant anglais avaient indéniablement exercé une influence importante sur les recherches de son admirateur écossais, l'entreprise de Maxwell avait une portée à laquelle les travaux de Faraday ne pouvaient pas prétendre. Maxwell se proposait, en effet, de révolutionner le traitement mathématique de l'électromagnétisme auquel Faraday, autodidacte dépourvu de culture mathématique, n'avait pas accès. Sur le plan des méthodes physico-mathématiques, les innovations de Maxwell avaient un lien beaucoup plus direct avec les travaux de Thomson qui, à partir de 1845, avait mis en relation les conceptions physiques de Faraday avec les méthodes mathématiques de la théorie du potentiel de Green ${ }^{84}$. Mais, à la différence de Thomson qui était demeuré agnostique quant à la validité

83. MAXWELL, 1873, p. x (c'est moi qui souligne).

84. Voir, sur ce sujet, WISE, 1981, et SмIтH \& WISE, 1989, chap. vII et viII. 
des conceptions physiques de Faraday, Maxwell entendait proposer dans son ouvrage une théorie mathématique fondée sur celles-ci ${ }^{85}$.

Le caractère particulièrement ambitieux de l'entreprise de Maxwell tenait au fait qu'il s'agissait, non pas de transmettre à ses lecteurs des connaissances acquises précédemment, mais, dans le même temps, de construire et d'exposer une nouvelle science de l'électromagnétisme. C'est ainsi qu'il faut interpréter une remarque de Maxwell, à propos de son livre, rapportée par John Larmor :

«A remark of his has been handed down [...] that the aim of his book was not to finally expound the theory for the world, but to educate himself by the presentation of a view of the stage he had reached ${ }^{86}$."

En rédigeant son traité, c'est à lui-même, aussi bien qu'à ses lecteurs, que Maxwell souhaitait enseigner une nouvelle façon de concevoir les phénomènes électromagnétiques. L'entreprise scientifique de Maxwell était donc indissociable de la nature pédagogique de l'ouvrage. Le traité permettait, en effet, de tenter une nouvelle mise en ordre des différents éléments théoriques de cette science en proposant un exposé qui parte de ses aspects les plus élémentaires pour progresser vers les plus complexes. L'ampleur de l'espace physique qu'offrait un livre de plusieurs centaines de pages permettait de développer une nouvelle pratique théorique de l'électromagnétisme comportant des notions, des méthodes et des valeurs méthodologiques nouvelles. Ainsi, loin d'être limitée à quelques chapitres isolés de l'ouvrage, la transformation de l'électromagnétisme se jouait sur l'ensemble du texte.

Une telle volonté de rupture était en partie justifiée par le contexte de la publication. En effet, le traité de Maxwell semblait consacrer une évolution théorique qui avait été à l'origine de la reconnaissance de l'électromagnétisme dans les universités britanniques. En situant son entreprise scientifique dans le prolongement des travaux de Faraday et de Thomson, Maxwell présentait son ouvrage comme l'émanation d'une nouvelle science britannique de l'électromagnétisme, fondée sur la notion de champ, qui devait se substituer à la science continentale «ordinaire », édifiée en France et poursuivie en Allemagne, et fondée sur la notion d'action à distance et la primauté du concept de charge électrique. Or, les travaux de Faraday et, surtout, de Thomson avaient fortement contribué, à travers leur rôle dans les succès de l'industrie télégraphique britannique, à faire

85. Cette attitude est exprimée dans la préface par une belle métaphore opposant le rôle d'un juge à celui d'un avocat, voir Maxweu, 1873, p. xIII ( $3^{c}$ éd., p. xu) : * I have therefore taken the part of an advocate rather than that of a judge, and have rather exemplified one method than attempted to give an impartial description of both. "

86. LARMOR, 1908, p. XIX; cité par EveriTt, 1978, p. 204. 
admettre dans les universités les progrès réalisés dans l'étude de l'électromagnétisme. La réforme de l'électromagnétisme entreprise par Maxwell était ainsi tacitement justifiée par la nécessité de consacrer les nouvelles méthodes théoriques employées dans la science et l'industrie télégraphique britanniques $^{87}$.

À partir du début des années 1880, l'influence des conceptions de Maxwell sur les recherches électromagnétiques menées en Grande-Bretagne et aux États-Unis témoigne de la spectaculaire réussite de son entreprise scientifique. Le décalage entre la publication du Treatise et les premiers signes tangibles de l'influence des conceptions de Maxwell correspond approximativement au laps de temps nécessaire pour que de jeunes physiciens formés à l'électromagnétisme par la lecture de l'ouvrage, bientôt appelés les " maxwelliens", produisent leurs premiers travaux. Conséquence des liens du Treatise avec le Mathematical Tripos et de la présence de Maxwell à Cambridge, un groupe important de ces «maxwelliens" avaient été formés à Cambridge : en particulier, le futur successeur de Maxwell à la tête du Cavendish Laboratory, Joseph John Thomson (18561940). Mais du fait de sa position unique parmi les ouvrages de langue anglaise traitant de l'électricité et du magnétisme, le Treatise exerça également son influence sur des jeunes hommes formés dans d'autres universités du Royaume-Uni ou des États-Unis : George Francis Fitzgerald (18511901) à Dublin, Oliver Lodge (1851-1940) à Londres ou Edwin Herbert Hall (1855-1938) à Baltimore. Le Treatise servit même à former un très brillant autodidacte : Oliver Heaviside $(1850-1925)^{88}$. Vers 1884, l'influence des conceptions de Maxwell sur les travaux de ces jeunes physiciens britanniques et américains était suffisamment profonde pour ne pas être ébranlée par le scepticisme affiché publiquement par William Thomson envers certains aspects essentiels de la théorie de Maxwell lors d'une célèbre série de conférences données à Baltimore ${ }^{89}$. On sait, enfin, que le Treatise permit la diffusion des conceptions de Maxwell dans des pays non anglophones : en particulier, l'Allemagne ${ }^{90}$ et la France ${ }^{91}$.

87. Le lien explicite entre les travaux de Faraday, Thomson et Maxwell et l'industrie télégraphique britannique est établi dans la préface de l'Electricity and magnetism de Fleeming Jenkin, voir supra, p. 535.

88. Pour une analyse de l'électromagnétisme à la fin du xix ${ }^{c}$ siècle, voir Buchwald, 1985. Pour un récit plus particulièrement centré sur les travaux et la personnalité de Fitzgerald, Heaviside et Lodge, voir HuNT, 1991. Il existe également une étude biographique de Heaviside : YAVETZ, 1995.

89. Pour l'opposition publique de Thomson à la théorie de Maxwell à partir de 1884, voir SMrTr \& WISE, 1989, chap. XIII. Pour la réaction des maxwelliens, voir aussi HUNT, 1991.

90. Darrigol, 1992.

91. Atten, 1992. 
L'étude du contexte institutionnel dans lequel eut lieu la publication du Treatise conduit donc à une nouvelle image de l'entreprise scientifique que l'ouvrage mettait en ouvre. Au moment de conclure, il est utile de présenter brièvement les perspectives d'enquête qu'une telle image paraît suggérer. L'étude des relations que le projet de Maxwell entretenait avec son contexte institutionnel tend à souligner les contraintes qui s'imposaient à l'écriture de l'ouvrage. La publication du Treatise s'inscrivait, en effet, dans un mouvement de reconnaissance institutionnelle des progrès réalisés dans l'étude de l'électricité et du magnétisme au cours des deux décennies précédentes. L'ouvrage devait donc intégrer ces apports théoriques dont Thomson avait été le principal artisan, mais on sait pourtant que le Treatise s'écarta suffisamment des normes établies par les travaux de Thomson pour que celui-ci rejette publiquement la théorie de Maxwell à partir de 1884. La rédaction du Treatise était donc traversée par une tension entre, d'une part, la nécessité de se conformer à l'orthodoxie de la physique britannique, représentée par la physique de Thomson, et, d'autre part, le souci de proposer une transformation de l'électromagnétisme beaucoup plus radicale que les innovations apportées par celui-ci. Il serait souhaitable de mieux cerner la manière dont Maxwell a tenté de concilier ces deux exigences. En d'autres termes, comment a-t-il cherché à révolutionner l'électromagnétisme tout en présentant cette entreprise comme un simple prolongement des travaux de ses prédécesseurs?

Franck ACHARD ${ }^{92}$

(juillet 1998).

92. Je tiens à remercier Éric Brian, Ernest Coumet et Robert Fox qui ont encadré ma recherche ainsi que Michel Atten, Michel Blay, Olivier Darrigol et Andrew Warwick qui m'ont fait bénéficier d'entretiens fort précieux. Je remercie également de son aide Peter Foden, archiviste à l'Oxford University Press. 


\section{BIBLIOGRAPHIE}

\section{Abreviation}

DSB : Dictionary of scientific biography, 1970-1980, éd. Charles Coulston GiLlispiE, New York, Charles Scribner's Sons.

\section{Sources primaires}

Arry (George Biddell), 1896, Autobiography of sir George Biddell Airy, éd. Wilfred Alry, Cambridge, Cambridge University Press.

JenkIn (Fleeming), 1873, Electricity and magnetism, Londres, Longmans, Green and $\mathrm{Co}$.

Maxwell (James Clerk), 1871, Theory of heat, Londres, Longmans, Green and Co.

MAXWELL (J. CL.), 1873, A treatise on electricity and magnetism, 2 vol., Oxford, Clarendon Press $\left(2^{\mathrm{e}}\right.$ éd. $1881,3^{\mathrm{e}}$ éd. 1891 , réimpr. de la $3^{\mathrm{e}}$ éd. New York, Dover, 1954).

MAXWELL (J. CL.), 1881, An elementary treatise on electricity, éd. William GARNETT, Oxford, Clarendon Press.

Maxwell (J. CL.), 1890, The Scientific Papers of James Clerk Maxwell, éd. William Davidson Niven, 2 vol., Cambridge, Cambridge University Press (réimpr. en 1 vol., New York, Dover, 1965).

Maxwell (J. CL.), 1990, Scientific Letters and papers of James Clerk Maxwell, vol. I : 1846-1862, éd. Peter M. Harman, Cambridge, Cambridge University Press.

Maxwell (J. CL.), 1995, Scientific Letters and papers of James Clerk Maxwell, vol. II : 1863-1873, éd. P. M. HaRman, Cambridge, Cambridge University Press.

Thомson (William), 1872, Reprint of papers on electrostatics and magnetism, Londres, Macmillan \& Co.

Thomson (William) \& TArT (Peter Guthrie), 1867, Treatise on natural philosophy, Oxford, Clarendon Press ( $2^{c}$ éd. en 2 vol., Cambridge, Pitt Press, 1879 \& 1883).

\section{Littérature secondaire}

ATten (Michel), 1992, Les Théories électriques en France, 1870-1900. La contribution des mathématiciens, des physiciens et des ingénieurs à la construction de la théorie de Marwell, thèse de doctorat, Paris, École des hautes études en sciences sociales.

Buchwald (Jed Z.), 1985, From Maxwell to microphysics. Aspects of electromagnetic theory in the last quarter of the nineteenth century, Chicago, University of Chicago Press. 
Campbell (Lewis) \& GarnetT (William), 1882, The Life of James Clerk Maxwell, Londres (réimpr. 1969, New York, Johnson Reprint).

CARDWELl (Donald), 1957, The Organization of science in England, Melbourne, Heinemann.

Chipman (Robert A.), 1976, "Jenkin, Henry Charles Fleeming ", DSB, vol. 7, p. 93-94.

DarRIGol (Olivier), 1992, "German expositions of Maxwell's Theory as documented by early German expositions of "Maxwell's Theory" ", Archive for the history of exact sciences, vol. 45, 3, p. 189-280.

EVERITT (C. W. Francis), 1978, « Maxwell, James Clerk », DSB, vol. 9, p. 198-230.

GaLison (Peter), 1983, "Re-reading the past from the end of physics. Maxwell's equations in retrospect $»$, Functions and uses of disciplinary histories, vol. VII, éd. Loren Graham, Wolf Lepenies et Peter Weingart, Dordrecht, D. Reidel Publishing Co.

Gooday (Graeme), 1990, «Precision measurement and the genesis of physics teaching laboratories in Victorian Britain », British Journal for the history of science, vol. 23, 1, p. 25-51.

HENDRY (John), 1986, James Clerk Maxwell and the electromagnetic field, Bristol, Hilger.

Hunt (Bruce), 1991, The Maxwellians, Londres, Cornell University Press.

KNotT (Cargill Gilston), 1911, Life and scientific work of Peter Guthrie Tait, Cambridge, Cambridge University Press.

LARMOR (John), 1908, « Obituary notices of fellows deceased : lord Kelvin », Proceedings of the Royal Society, vol. LXXXI A.

MOYER (Donald Franklin), 1977, « Energy, dynamics, hidden machinery. Rankine, Thomson and Tait, Maxwell ", Studies in history and philosophy of science, vol. 8,3, p. $251-268$.

Moyer (D. F.), 1978, « Continuum mechanics and field theory. Thomson and Maxwell ", Studies in history and philosophy of science, vol. 9, 1, p. 35-50.

SCHAFFER (Simon), 1992, "Late Victorian metrology and its instrumentation. A manufactory of Ohms ", in Invisible Connections. Instruments, institutions and science, éd. Robert Bud et Suzan E. Cozzens, Bellingham, WA, SPIE Optical Engineering Press.

SchAFFER (S.), 1995, «Accurate measurement is an English science », in The Values of precision, éd. M. Norton WISE, Princeton, NJ, Princeton University Press.

Schuster (Arthur), 1910, A history of the Cavendish Laboratory, 1870-1910, Londres, Longmans.

SIEGEL (Daniel), 1991, Innovation in Maxwell's electromagnetic theory. Molecular Vortices, displacement current, and light, Cambridge, Cambridge University Press.

Simpson (Thomas K.), 1970, « Some observations on Maxwell's Treatise on electricity and magnetism. On the role of the "Dynamical theory of the electromagnetic field" in part IV of the Treatise ", Studies in history and philosophy of science, vol. 1, 3, p. 249-263.

SMrTH (Crosbie), 1978, "A new chart for British natural philosophy. The development of energy physics in the nineteenth century ", History of science, vol. 16, p. 231-279.

SMITH (C.) \& WISE (M. N.), 1989, Energy and empire. A biographical study of lord Kelvin, Cambridge, Cambridge University Press.

Sutcliffe (Peter), 1978, The Oxford University Press. An informal history, Oxford, Oxford University Press. 
Sviedrys (Romualdas), 1970, "The rise of physical science at Victorian Cambridge ", Historical Studies in the physical sciences, vol. 2, p. 127-151.

Sviedrys (R.), 1976, "The rise of physics laboratories in Britain ", Historical Studies in the physical sciences, vol. 7, p. 405-436.

Szabadvary (Ferenc), 1973, « Esson, William », DSB, vol. 4, p. 411-412.

Thompson (Silvanus Phillips), 1910, The Life of William Thomson, baron Kelvin of Largs, Londres, Macmillan.

Thomson (Joseph John), éd., 1931, James Clerk Maxwell. A commemoration volume, 1831-1931, Cambridge, Cambridge University Press.

WILson (David B.), 1982, « Experimentalists among the mathematicians. Physics in the Cambridge Natural Tripos, 1851-1900 ", Historical Studies in the physical sciences, vol. 12, 2, p. 325-371.

Wise (M. Norton), 1979, «William Thomson's mathematical route to energy conservation. A case study of the role of mathematics in concept formation ", Historical Studies in the physical sciences, vol. 10, p. 49-83.

WISE (M. N.), 1981, « The flow analogy to electricity and magnetism, Part I : William Thomson's reformulation of action at a distance ", Archive for the history of exact sciences, vol. 25, 1, p. 19-70.

YAveTZ (Ido), 1995, From obscurity to enigma. The work of Oliver Heaviside, 18721889, Bâle/Boston/Berlin, Birkhäuser. 